\title{
EL MÉTODO MONTESSORI EN LA EDUCACIÓN INFANTIL ESPAÑOLA: LUCES Y SOMBRAS
}

\section{The Montessori method in the Spanish early childhoob education: lights and shadows}

\author{
Carmen SANCHIDRIÁn BLANCO \\ Universidad de Málaga \\ Correo-e: sanchidrian@uma.es
}

Recepción: 9 de junio de 2020. Envío a informantes: 13 de junio de 2020 Aceptación definitiva: 30 de julio de 2020

Resumen: En este artículo se revisa la Introducción, difusión y aplicación de la pedagogía de Montessori en España en tres etapas. Durante la primera (I9I4-I936) se editan en español muchas de sus obras, se realizan las primeras experiencias y la misma Montessori pasa años en nuestro país; en la segunda (franquismo), tras dos décadas de rechazo frontal a toda la Escuela Nueva, Montessori fue encontrando su pequeño lugar, y, por último, en la España democrática veremos una cierta diversidad e incluso confusión en torno a la aplicación de sus métodos. La vida y la obra de Montessori han estado ocultas por escritos hagiográficos y es preciso estudiarlas sin perder de vista su contexto, su educación, sus creencias y sus intereses; solo así se podrá distinguir a los que mantienen sus principios pedagógicos de los que intentan vender cosas diversas en su nombre.

Palabras clave: método Montessori; Historia de la Educación; Educación infantil; Prácticas educativas; España.

AвSTRACT: This article analyses the introduction, dissemination and application of Montessori pedagogy in Spain in three stages. It was during the first period (19I41936) when many of her works were published in Spanish, the first experiences took place and Montessori herself spent years here. In the second stage (I936-I975), after two decades of frontal rejection of the entire New School, Montessori found her place. And finally, in the third stage, coinciding with democracy in Spain, a certain diversity and even confusion regarding the application of its methods can be identified. Montessori's life and works have been hidden by hagiographic writings. It is 
necessary to approach her taking into account her context, education, beliefs and interests. Only by doing so will it be possible to distinguish those who apply her theories from those who are just trying to sell something different on her name.

KEY words: Montessori Method; Educational History; Early Childhood Education; Educational Practices; Spain.

\section{Introducción}

M

ARIa Montessori (I870-I952) tiene en Google 5.680.00o entradas frente a 3.900.000 de John Dewey, I09.000 de Ovide Decroly o 28.000 de Célestin Freinet, poniendo siempre nombre y apellidos entre comillas para evitar muchas otras que nada tengan que ver con ellos ${ }^{1}$. Es evidente que Montessori en España es un nombre que hoy suena a progresista, alternativo, elitista, aunque pocos hayan leído sus obras para ver qué es eso del método Montessori. El número de colegios que dicen seguir este método crece anualmente, especialmente en algunos países, aunque es difícil tener cifras exactas, como veremos luego.

Se ha dicho, escrito y repetido tanto sobre Montessori que hablar sobre ella es un reto $^{2}$. En primer lugar, porque se tiene la sensación de que es difícil decir algo que no se haya dicho y porque existen muchos prejuicios y estereotipos en torno a lo que es o no es, lo que dijo o no dijo, Montessori, sin haberla leído. En los últimos años, sin embargo, han ido apareciendo estudios ${ }^{3}$ que, con fuentes inéditas y relevantes, nos permiten acercarnos a nuevas interpretaciones tanto de su vida como de su obra, algunas de las cuales bajan a Montessori «respetuosamente» de su pedestal, tal como se decía en el folleto publicitario del libro de Schwegman ${ }^{4}$ ya citado. Y, en segundo lugar, porque hoy Montessori no solo es

Búsquedas realizadas el 2 de mayo de 2020.

En Montessori Life, revista oficial de la AMS, American Montessori Society. hay tres recopilaciones de artículos, todos en inglés, publicados en revistas ajenas a centros o asociaciones Montessori que incluyen información sobre ella y/o sobre su método: BAGBY, J. H.: «Montessori Education and Practice: A review of the Literature, 1996-2006», Montessori Life, I (2007), pp. I-8; BAGBY, J. H. y Jones, N. A.: «Montessori Education and Practice: A review of the Literature, 2007-2009", Montessori Life, 2I-4, pp. 44-48; Bagby, J.; Wells, K.; Edmonson, K. y Tompson, L.: «Montessori Education and Practice: A review of the Literature, 20IO-2013», Montessori Life, Spring (20I4), pp. 32-4I.

Depaepe, M.: «Montessori privée du piédestal? Compte rendu de deux études récentes aux Pays-Bas», Paedagogica Historica, XXXV (2) (1999), pp. 425-431. Marc Depaepe daba cuenta ahí de dos obras que pretendían ofrecer una imagen de Montessori alejada de las habituales mitificaciones: Schwegman, M.: Maria Montessori, I870-1952 Kind van haar tijd. Vrouw vande wereld, Amsterdam, Amsterdam University Press, 1999, y LeEnders, H.: Montessori en fascistisch Italië. Een receptiegeschiedenis, Baarn, Uitgeverij Intro, 1999. A estas dos publicaciones hay que añadir Foschi, R.: Maria Montessori, Barcelona, Octaedro, 20I4. Foschi aborda con detalle aspectos poco tratados antes como sus difíciles relaciones con Talamo, primero, y luego su interés en el catolicismo y en el fascismo italiano a partir de documentos conservados en el Archivo del Generalato de las Franciscanas Misioneras de María y en el Archivo Central del Estado (ACS), ambos en Roma.

4 http://www.letterenfonds.nl/en/book/35/maria-montessori-1870-1952. 
EL MÉTODO MONTESSORI EN LA EDUCACIÓN INFANTIL ESPAÑOLA:

LUCES Y SOMBRAS

CARMEN SANCHIDRIÁN BLANCO

una marca, sino una marca que vende, y que vende de todo: por supuesto, materiales didácticos, colegios, formación, publicaciones, etc., pero también muebles para habitaciones infantiles y de $\operatorname{todo}^{5}$ (hay blogs, charlas TED y publicaciones de diverso alcance $)^{6}$. Y, por tanto, hay que tener cuidado no vaya a ser que bajo la marca «Montessori» nos quieran vender otras cosas.

Montessori es uno de los personajes más conocidos especialmente en el ámbito de la educación infantil. Ella misma fue su mejor agente publicitario y dedicó la mayor parte de su vida a difundir sus ideas, su método, sus materiales ${ }^{7}$. Contribuyó directamente a la elaboración de su propia biografía, sin elaborar una autobiografía, a través de datos biográficos y de comentarios y valoraciones acerca de sus aportaciones que incluía en sus obras, por ejemplo, cuando describe la historia de su método, dando de sí misma una imagen que luego no coincide con la realidad en ciertos aspectos ${ }^{8}$. Muchos de esos datos se han repetido hasta la saciedad y se siguen manteniendo a pesar de haber sido corregidos. Por ejemplo, ella contaba que estudió Medicina en la Universidad de Roma, graduándose en i896, y que fue la primera mujer en lograrlo en Italia y todas las biografías así lo repetían. En 1999 se refutó ese mito demostrándose que no había sido la primera ${ }^{9}$, pero sigue apareciendo ese dato casi todos los textos ${ }^{10}$. De hecho, la primera mujer que obtuvo el título en Medicina y Cirugía en Italia fue Ernestina Paper en I877 en Florencia, año en que abrió una consulta donde atendía a mujeres y niños.

En 2020 se celebra el 150 aniversario del nacimiento de Montessori. Han pasado más de cien años desde que Montessori abrió sus primeras Casas de los Niños en 1907 en San Lorenzo, Roma, y en 2015 se cumplieron cien años de la publicación en castellano de su obra más importante, su Método de la Pedagogía Científica, la obra que mejor recoge sus teorías sobre educación. En este siglo, la educación ha cambiado más que en todos los siglos anteriores juntos. Esta afirmación, que es cierta en sí, lo es más aún cuando la aplicamos a la hoy llamada educación infantil

Muñoz, Beatriz: «Ikea y Montessori», [Post en un blog, I2 de mayo de 20I5]. Recuperado de https://tigriteando.com/ikea-y-montessori/ [consultado 14-5-2020]; TÉBAR, Cristina: «¿Existe la «ropa Montessori»? [Post en un blog, 22 de octubre de 2019]. Recuperado de https://www.montessoriencasa.es/existe-la-ropa-montessori/ [consultado Io-5-2020]: se recomienda aquí «Intentar evitar ropa, mochilas, etc., con personajes de fantasía, violencia...».

6 Por ejemplo, una novela gráfica sobre su vida; Ronda, M.: María Montessori. Una vida para los niños, Barcelona, Bruguera (Ediciones B), 2020; un libro para aplicar Montessori en casa, MUÑoz, B.: Montessorizate. Criar siguiendo los principios Montessori, Barcelona, Grijalbo, y un volumen de la colección Mujeres extraordinarias: Maria Montessori La maestra que enseñaba a través del juego, Barcelona, Emse Edapp D.L. y Editorial Salvat, 2019.

Sanchidrián Blanco, C.: «Introducción», en Montessori, M.: El método de la Pedagogía científica, Madrid, Biblioteca Nueva, Colección Clásicos de la Pedagogía, 2003, pp. 9-8I.

${ }_{8}$ Cfr. Montessori, M.: El método de la Pedagogía científica, op. cit., capítulos i y 2. En realidad, por ejemplo, no trabajó de forma continuada con los niños (a pesar de la imagen que ella misma pretende ofrecer de su tarea); ejerció como docente en el sentido de explicar, enseñar y difundir su teoría y su método educativos a otros maestros.

9 Cfr. Schwegman, M.: Op. cit.

ro De hecho, sigue siendo habitual que se diga que fue la primera en vez de que fue una de las primeras. No es un dato importante en sí mismo, pero sí revela un rasgo de su personalidad. 
o, en general, a la educación de los niños antes de la edad escolar. Y esto es así, en primer lugar, por el cambio en el concepto de niño, y ahí todos los autores coinciden en la contribución de Montessori no ya al descubrimiento del niño, pero sí a la consideración de la infancia como un período especial dentro de la vida del ser humano en la línea de lo planteado por Rousseau. Y, en segundo lugar, porque la práctica generalización de la educación formal de los niños de cuatro y cinco años (y también de tres en España) ha motivado un fuerte interés por los métodos de educación infantil.

En estos más de cien años, se han introducido cambios en las escuelas Montessori y hemos de reconocer que una de las características del movimiento montessoriano, como en cualquier propuesta que pretenda durar, ha sido, precisamente, su capacidad de adaptación, aunque, cuanto mayor es esta, menor es la fidelidad a la teoría primitiva. Hay que admitir, por ejemplo, que hoy, cuando se pretende poner el acento en todo el sistema educativo en el aprendizaje más que en la enseñanza, Montessori, que tanto insistía en el autoaprendizaje, puede resultar distinta y reveladora. Estamos, pues, frente a un método centenario, no frente a una «novedad», aunque a menudo los colegios Montessori se «presenten» como innovadores, como lo último en educación, y esto habría que matizarlo. Pero, en estos cien años, el método Montessori se ha convertido en un clásico, en el sentido de que podemos ver en él nuevos matices e interpretarlo a la luz de los nuevos conocimientos ${ }^{11}$.

En este artículo veremos la Introducción, difusión y aplicación de la pedagogía de Montessori en España en tres etapas: la primera coincide con el comienzo de su deambular por el mundo y del proceso de internacionalización de su obra, de 1914 a 1936: es cuando se editan en español muchas de sus obras, se realizan las primeras experiencias y la misma Montessori pasa años en nuestro país; la segunda, en el franquismo, cuando, tras un rechazo frontal a toda la Escuela Nueva, Montessori se fue haciendo un hueco, y, por último, Montessori en la España democrática, donde se aprecia una cierta diversidad e incluso confusión en torno a la aplicación de sus métodos.

\section{Llegada de Montessori a España (I9II-I936)}

En este período comenzó lo que Foschi denomina el infinito deambular nómada de Montessori. Este autor la considera un ejemplo de «cerebro en fuga», en el sentido de que fue más valorada fuera que dentro de su país natal. Fueron unos años difíciles para ella, marcados por sucesivas rupturas, pues rompió con Talamo, con el Instituto Romano de Bienes Inmuebles (IRBS) y con las Franciscanas Misioneras de María (FMM) y, además, su propuesta metodológica «perdió» a

"Sanchidrián-Blanco, C.: «La pedagogía de Montessori en el siglo xxi», en Pallarés, M. (coord.): El pensamiento pedagógico del siglo XX y la acción educativa del siglo XXI, Barcelona, Octaedro, pp. 49-70. 
EL MÉTODO MONTESSORI EN LA EDUCACIÓN INFANTIL ESPAÑOLA:

LUCES Y SOMBRAS

CARMEN SANCHIDRIÁN BLANCO

nivel nacional frente a la de las hermanas Agazzi. Después, en 1928, empezaría su colaboración con el fascismo italiano que también terminaría en una ruptura.

En I9I2 se celebró en Roma el I Curso Internacional Montessori y a partir de ahí empezó a visitar diversos países en los que pronto se fue extendiendo su método. Dentro de ese nomadismo, pasó temporadas en España, en concreto en Barcelona, que hasta la Guerra Civil la consideró ciudadana suya.

Veamos algunos datos sobre el comienzo de ese nomadismo que prácticamente mantuvo de por vida ${ }^{12}$. Recordemos que en 1907 se había inaugurado la primera Casa de los Niños en el Barrio de San Lorenzo de Roma, promovida por Eduardo Talamo, director del IRBS; en 1908 se inauguran las Casas de los Niños de la Sociedad Humanitaria de Milán y en 1909 la Casa de los Niños de Via Giusti del Generalato de las FMM. Montessori se acercó entonces más al catolicismo y las FMM posibilitaron la apertura de más Casas. Ese mismo año se publicó la Pedagogía científica con el apoyo de Alice y Leopoldo Franchetti. Sin embargo, poco después, en I913, se produjo la ruptura de Montessori con Roma, con el IRBS y con las FMM, y Montessori comenzó sus continuos viajes buscando el apoyo que no encontró en su país. Esas sucesivas rupturas explican gran parte de la vida de Montessori, por lo que conviene conocerlas aunque se ha hablado poco hasta ahora de ellas ${ }^{13}$.

Las relaciones entre Montessori y Eduardo Talamo, quien propuso a Montessori hacerse cargo de una institución educativa en el barrio de San Lorenzo de Roma, siendo así como surgieron las Casas de los Niños, fueron pronto conflictivas. La denominación Casa de los Niños se convirtió pronto en Italia en sinónimo de lugar educativo moderno y de vanguardia, pero «a Montessori le interesaba sobre todo desarrollar un método pedagógico innovador, reconocido por la comunidad científica; Talamo, en cambio, tenía objetivos higienistas y, en última instancia, se planteaba aunar los intereses de sus accionistas, unos ideales humanitarios y la ingeniería civil» ${ }^{14}$.

La polémica entre ambos surgió quizá porque Montessori no quería que su método se identificara con una rama del IRBS. En una carta de Talamo a Olga Lodi, periodista feminista amiga común de Montessori y Talamo, este muestra su extrañeza ante la postura de Montessori

Una cosa es el método Montessori, otra la Casa de los Niños. El método Montessori, genial descubrimiento al que es de justicia atribuir grandes honores, refleja puramente la pedagogía infantil; la Casa de los Niños, o sea, la escuela en casa, por el nombre y por la sustancia, fue en cambio creada por el instituto Romano de los Bienes Inmuebles, el cual quiso ponerla en práctica en sus casas populares, precisamente porque, como factor de civilización del pueblo, formaba parte de la

${ }_{12}$ En esta breve entrevista realizada a Montessori en 1947, aprovecha para decir los países recorridos últimamente y las actividades desarrolladas. «Le nostre interviste: A coloquio con Maria Montessori», La Settimana Incom, o0058 del I3/05/1947. Archivio Storico LUCE. Disponible en https://www.youtube.com/watch?v=-BL5q8MSNh8.

${ }_{13}$ Seguiremos la descripción de los hechos que presenta FOSCHI, R.: Op. cit., pp. 47-77.

${ }^{14}$ Ibidem, pp. 57-58. 
transformación moderna de la vivienda. [...] Entre el método Montessori y la Casa de los Niños no hay más que una cosa en común, esto es, que tras sugerencia de una amable señora a la que me dirigí para que me aconsejara, en lugar de adoptar, en cuanto a la parte pedagógica, los habituales métodos hasta entonces en boga en las Guardería infantiles, accedí a dejar experimentar en gran escala los excelentes métodos de la ilustre profesora Montessori ${ }^{15}$.

Por tanto, entre 1908 y 1909 se rompió la colaboración Talamo-Montessori, aunque esta publicó en 1909 su Método de la Pedagogía científica describiendo su experiencia con relación a las Casas de los Niños, reconstruyéndola como si hubiese sido el resultado de un trabajo científico y experimental. Montessori dijo que tras su ruptura con Talamo se le había impedido incluso entrar en las Casas de los Niños de San Lorenzo. Talamo no entendía el rechazo de Montessori a que se utilizara su método y le había amenazado con un litigio:

En cuanto a retirar los métodos, que haga lo que quiera; perderá más ella cuando se diga que los B. Inmuebles la han proscrito de sus C. de los Niños; a pesar de que no sé cómo se pueden retirar los métodos, toda vez que se venden los aparatos de la enseñanza en la Humanitaria, que pasa a su inventora el $20 \%$ del precio. Sería como si quien hubiese publicado un libro de historia o de gramática que está a la venta en librerías, pretendiese que quien lo estudie esté obligado a tomar al autor como profesor, de lo contrario no se estudia ${ }^{16}$.

Su ruptura con la Humanitaria de Milán, que construía los materiales a Montessori, fue otro punto de conflicto. Tras terminar su colaboración con el IRBS, comenzó, entre 1908 y I9Io, un período de fuerte colaboración entre Montessori y los católicos: en ı9ıo, en Via Giusti ı2, en la casa generalicia de las FMM, fundó una Casa de los Niños y desde i9ız se impartieron los primeros cursos nacionales sobre el método.

En ese momento, Montessori planteó su movimiento pedagógico como una asociación pedagógica femenina y católica. Hay borradores de las reglas que las primeras montessorianas deberían haber seguido en esa hipotética congregación: «Exteriormente no debería haber ningún signo de nuestro estado real; con todo creo firmemente que no se puede servir a dos señores, y que quien viene con nosotros debe dejar el mundo y la familia» ${ }^{17}$. El proyecto de congregación pedagógica y religiosa incluía, además, una profesión de fe católica y los deberes morales e intelectuales a los que las maestras debían atenerse. Así se explica algo que se puso de relieve en algunas biografías, el círculo de las primeras alumnas se asemejaba mucho a un pequeño cenáculo agrupado bajo la protección de «María» ${ }^{18}$.

is Citado en ibidem, p. 6o.

16 Citado en ibidem, pp. 58-59.

${ }_{17}$ Ibidem, p. 68. Foschi cita un librito conservado en el archivo de Via Giusti, escrito probablemente por Montessori, donde está el borrador de la «regla» de esa supuesta congregación.

${ }_{18}$ Kramer, R.: Maria Montessori: A biography, New York, Da Capo Press, 1988, 2. ${ }^{a}$ ed., p. 179. Cit. por Foschi, R.: Op. cit., p. 69. 
EL MÉTODO MONTESSORI EN LA EDUCACIÓN INFANTIL ESPAÑOLA:

LUCES Y SOMBRAS

CARMEN SANCHIDRIÁN BLANCO

Este acercamiento tan sui generis al catolicismo chocaba con los ambientes donde se había formado y se traducía, por ejemplo, en momentos disciplinados de plegarias en las Casas de los Niños abiertas por las FMM que no había en las del IRBS. Su tentativa de demostrar que su pedagogía no solo no era peligrosa sino que estaba profundamente ligada al catolicismo, representaba probablemente una actitud «oportuna y autoconservadora» ${ }^{19}$.

Los documentos conservados en Via Giusti confirman el fracaso de este intento de acercamiento a la Iglesia católica a través de las FMM y de Agostino Gemelli (médico y franciscano, fundador de la Universidad Católica del Sagrado Corazón de Milán) que terminó también con una ruptura como vemos en esta cita del periódico Sentinella Antimodernista:

Tomando cuanto hay de bueno en el método de Montessori, las Franciscanas Misioneras de María han sabido cumplir en pocos meses nuevos prodigios. Han conseguido que niños de 3 a 6 años se convirtiesen en modelos, dóciles, graciosos y activos, dejándoles totalmente libres de cualquier constricción. [...] Lo que no creemos es esto: que el mucho bien hecho por las Hermanas franciscanas de Milán, observado por el P. Gemelli, se deba exclusivamente al método Montessori que creemos que choca contra el método tradicional cristiano de educación, contra el sentido común y que parece algo absurdo. [...] Montessori quiere que al niño se le deje completa libertad en sus actos y en la manifestación de su naturaleza. [...] ¿qué es este culto exagerado de la independencia y de la libertad llevado hasta el rechazo del premio del bien y del castigo del mal ?20.

Por tanto, entre I910 y 1913 el método se aplicó por las FMM según una perspectiva católica y Montessori y sus más estrechas colaboradoras habían pensado en constituirse incluso en congregación religiosa. Pero, desde el punto de vista público, Montessori era vista como laica y tuvo dificultades para ser aceptada por el ambiente católico al que se estaba acercando a través de las FMM. Las críticas del ámbito católico estaban en la línea con las de la pedagogía académica y así, entre I9I4 y I9I5, se cerró Via Giusti y terminó la colaboración con las FMM ${ }^{21}$.

Hubo otra razón de peso que empujó a Montessori fuera de su país. En I9I4 se creó una real comisión para examinar y evaluar los métodos más utilizados en Italia y se discutió acerca de las metodologías pedagógicas de Froebel, Agazzi y Montessori. En el informe final se abogaba por una orientación didáctica en la línea de las hermanas Agazzi. La principal crítica a Montessori era que favorecía al niño urbano de cierta clase y entorno que no podía ser considerado el representativo de la nueva Italia. El niño montessoriano se orientaba a la sociedad industrial que quería pasar sobre la sociedad agrícola, rural. Los motivos para elegir el método Agazzi como más adecuado para la reorganización del sistema italiano era

is Foschi, R.: Op. cit., p.72.

20 El fragmento S.A.C.: «P. Gemelli, il metodo Montessori e le Francescane Missionarie di Milano", Sentinella Antimodernista, I, I2 (I9I2), pp. I7I-I74. Citado por FosCHI, R.: Op. cit., pp. 72-73.

${ }_{21}$ Pueden verse más textos en torno a esta ruptura en Foschi, R.: «Science and culture around the Montessori's first 'children's houses' in Rome (1907-1915)", Journal of History of the Behavioral Sicences, 44, 3 (2008), pp. 238-257. 
que se adaptaba mejor ética y culturalmente al niño italiano, aunque no tuviera atributos específicos de italianidad. El informe de esta comisión fue decisivo para relegar el método Montessori fuera del sistema académico ${ }^{22}$.

Tras estos acontecimientos comenzaría la internacionalización de su obra ${ }^{23}$. Su método no fue aceptado por la pedagogía académica italiana del momento quizá porque la idea de dejar en libertad al niño y el fomento de la independencia, ajenos a premios y castigos, no se entendía, o no se podía aceptar, y se rechazaba de base. Por ello comenzó a ofrecer, y se siguen ofreciendo, cursos privados de formación de maestras y a gestionar la fabricación y venta de materiales didácticos exclusivos y a medida del niño creando así su propia marca: «Probablemente fue la capacidad empresarial de Montessori la que fascinó a los estadounidenses quienes, después de 1913, adoptaron e hicieron famoso tanto el método como a su inventora ${ }^{24}$. Sin embargo, de ese país vendrían también algunas de las críticas más serias: poco original, materiales caros, excesivamente mecánico, formal y limitado, excesivamente libertario e individualista... ${ }^{25}$.

Todo esto explica el deambular de Montessori tras la Primera Guerra Mundial, con «el objetivo explícito de encontrar apoyos para la construcción de una asociación internacional para la mejora de la humanidad por medio de la educación y la valorización del niño» ${ }^{26}$. A partir de ahí, rompió los lazos con Italia, aunque intentó retomarlos a través de su relación con Mussolini en los años veinte.

Los planteamientos pedagógicos de Montessori llegaron pronto a España vinculados al pedagogismo noucentista, como ya se ha explicado en otras oca-

22 Giardiello, P.: Pioneers in Early Childhood Education: The Roots and Legacies of Rachel and Margaret McMillan, Maria Montessori and Susan Isaacs, New York, Routledge, 2013, pp. 86-87.

${ }^{23}$ A finales de 1913 fue por primera vez a Estados Unidos para dar conferencias (volvió de nuevo en I9I5 y 1917). Desde el principio, empezaron a abrirse escuelas Montessori por todo el país y a aparecer artículos de entusiastas de su trabajo en la prensa, así como críticas de profesionales que abogaban por las teorías psicológicas y educativas del momento. Es curioso constatar que el movimiento, igual que se difundió, se apagó, de forma que en I9I8 aparecen escasas referencias al mismo o a estas escuelas. Schwegman (1999) puso de manifiesto que Montessori intentó, mediante todo tipo de intrigas, mantener el monopolio de su material, que solo podía ser producido con su autorización, lo que la colocó en una situación incómoda en Estados Unidos, y ejercer una especie de dictadura montessoriana. Sin embargo, en la década de 1970 se produjo un fuerte impulso realizándose numerosas experiencias en torno al método que han dado lugar a una bibliografía fuertemente elogiosa. La descripción, recogida por Sizaire, que de Montessori hace Lanternier, seguidora suya que tenía junto a su marido una escuela Montessori en Estados Unidos, refleja bien el ambiente que rodeaba a Montessori en sus viajes: «Bella, elegante y, sobre todo, desprendía una incré́ble fuerza vital, al tiempo que parecía un tanto inaccesible, como si estuviera en otro planeta. Crucé mi mirada con la suya, me senté y me puse a llorar..., recuerda Fanny Lanternier. Me sentía como comprometida, como ligada a ella». Fanny y su marido Michel habían fabricado el material para la escuela; asistieron al Congreso Internacional de San Remo y estaban subyugados por ella, como se ve en esa cita. Sizaire, A.: M. Montessori. La educación liberadora, Bilbao, Desclée de Brower, I995, p. 72.

${ }_{24}$ Foschi, R.: Op. cit., p. 82.

${ }_{25}$ Pueden verse algunas de las críticas que le hicieron a Montessori Dewey, Kilpatrick, Claparède y Radice en Sanchidrián Blanco, C.: Op. cit., pp. 48-58.

26 Idem. 
EL MÉTODO MONTESSORI EN LA EDUCACIÓN INFANTIL ESPAÑOLA:

LUCES Y SOMBRAS

CARMEN SANCHIDRIÁN BLANCO

siones $^{27}$, de la mano de Joan Palau Vera ${ }^{28}$, quien veía que el método Montessori encajaba con los objetivos de renovación social del momento y terminó siendo la realización más emblemática de la Mancomunidad de Cataluña. Por esto, la presencia de Montessori en España hay que enmarcarla, por una parte, en el momento vital que estaba viviendo Montessori necesitada del reconocimiento exterior que no encontraba en su país y, por otra, en el movimiento de renovación pedagógica que tuvo lugar en Cataluña, que estuvo ligado a este método no slo durante el primer tercio de este siglo, sino también, aunque ya en menor grado, tras la Guerra Civil ${ }^{29}$.

La revista Feminal había publicado, en I9II, la primera noticia acerca de las teorías de Montessori y al año siguiente la Revista de Educación explicaba el método más ampliamente. Palau era un buen conocedor de las instituciones pedagógicas innovadoras como la Institución Libre de Enseñanza, l’École des Roches o Abbotsholme, y había fundado dos kindergarten froebelianos llamados Montd'Or, el primero en Pedralbes, Barcelona, y el segundo en Tarrasa. Tras sus ensayos froebelianos se interesó por el montessorianismo, divulgó su sistema y tradujo la mayor parte de sus obras ${ }^{30}$. Palau fue pensionado para conocer las Case dei Bambini en Roma y luego el Consell d'Investigació Pedagógica de la Diputación de Barcelona le encargó ensayar experimentalmente el método en una clase de párvulos de la Casa de Maternidad y Expósitos, con alumnos acostumbrados al clásico parvulario de gradas. Este primer ensayo, asumido por Montessori tras el cierre de Via Giusti, contó con Anna Maccheroni, colaboradora de Montessori desde el primer momento, y con los padres y monjas vicencianos (san Vicente de Paul); comenzó en enero de I9I4, duró I4 semanas y es interesante porque permite ver el cambio experimentado por esos niños que se planteaba como similar al

27 Sobre Montessori en España, $c f r$. Sanchidrí́n Blanco, C.: Op. cit., pp. 23 -28.

28 Comas Rubí, F. y Sureda, B.: «The Photography and propaganda of the Maria Montessori method in Spain (I9II-1931)", Paedagogica Historica, 48, 4, I (2012), pp. 571-587.

29 Gracias a la preocupación educativa de personas y organismos catalanes como el Ayuntamiento, la Diputación y la Mancomunidad, se abrieron nuevos centros y se vivió intensamente la necesidad de renovación social, en general. Recordemos a pedagogos como Juan Bardina, Juan Palau, Rosa Sensat, Alejandro Galí o Pedro Vergés; centros como la Escuela del Bosque y la Escuela del Mar; los cursos de perfeccionamiento organizados por la Escola d'Estiu, y las ideas y experiencias pedagógicas tanto en torno a Montessori como a Claparède, Dewey, Decroly y Freinet. Todo ello formó un movimiento popular que situó a Cataluña en el primer lugar de España en renovación pedagógica. La primera Escola d'Estiu fue organizada en I9I4 por la Diputación de Barcelona. La dirigió Eladi Homs y se dedicó a difundir el método Montessori. Estas escuelas de verano para maestros se desarrollaron desde I9I4 a I923 y desde 1930 hasta la guerra. El programa de los actos celebrados en Girona en abril de 2014 con motivo del centenario de la primera escuela Montessori en España, organizados por la Asociación Montessori Española (AME), puede verse en https://montessoriparatodos.es/blog/wp-content/uploads/20I4/o2/Programa-Centenario-Montessori-Espa\% $33 \%$ Bra.pdf.

${ }_{30}$ La traducción y publicación de sus obras en castellano fue relativamente fluida a medida que iban apareciendo los originales, pero la reedición de estas obras no lo ha sido. En los últimos cuarenta años solo se han reeditado Manual práctico del todo Montessori e Ideas generales sobre mi método, ambos reunidos bajo el título Ideas generales sobre el método. Manual práctico, en 1994 por la editorial CEPE, y la primera edición de El método de la Pedagogía científica en 2003 por Biblioteca Nueva, ya citado. 
descrito por Montessori con relación a la primera Casa dei Bambini en 1907. De la dirección de esta escuela se encargó Mercè Climent, una de las maestras pensionadas para asistir al curso de Roma ${ }^{31}$.

En estos años la educación religiosa era el centro de la experiencia educativa. La relación de Montessori con el contexto católico tuvo siempre altibajos, «con nuevos acercamientos y repentinas rupturas. Probablemente la religión, en la óptica montessoriana, estuvo siempre filtrada por la luz de la experiencia teosófica de la que no renegó jamás» ${ }^{32}$. Ella misma indicaba que la formación religiosa en las Casas de los Niños, basada en criterios pedagógicos por medio de la adopción de materiales apropiados y el desarrollo de ritos litúrgicos, tuvo su origen en I9I5, en España, donde era una finalidad fundamental la enseñanza de la religión católica y como continuación de su experiencia en Via Giusti:

Cuando empezamos la educación religiosa en Barcelona, en la escuela Modelo Montessori, las líneas de conducta fueron al principio discutidas largo y tendido [...] El P. Casulleras de los sacerdotes de las Misiones de San Vicente de Paúl [...] todavía no sabía nada de mis Casas de los Niños en Roma [...] en rgro tuvo conocimiento por casualidad de mi libro en el que las describía. Al P. Casulleras le pareció providencial la coincidencia de los nombres, y leyendo la descripción de mi método lo juzgó apropiado para sus Casas de los Niños. Enseguida fue a hablarlo con el Capellán de la Casa de la Maternidad de Barcelona, el P. Clascar [...] uno de los fundadores del Instituto de Estudios Catalanes. Enseguida se pusieron de acuerdo en aplicar mi método a los niños de la Maternidad; y así lo hicieron e igualmente sucedió con todos los Institutos de huérfanos de las Islas Baleares. A pesar de que aquellos Padres no me conocían e ignoraban que yo era católica, y a pesar de que en el libro no hacía ninguna profesión de fe religiosa, les pareció a ellos que mi método era católico en su misma substancia ${ }^{33}$.

La misma Montessori explica cómo se adaptó su método y se preparó el ambiente para esta enseñanza, el mobiliario, decoración, papel del sacerdote y sus explicaciones y los trabajos campestres en la educación religiosa (cultivo de trigo y vides y siega y vendimia) $)^{34}$. Entre 1922 y 1923 Montessori publicó algunos libros sobre educación del niño mediante la religión y el rito de la misa, pero su luna

31 Comas Rubí, F. y Sureda, B.: «La prensa ilustrada y su aportación a la publicidad del método de Maria Montessori en España en el primer tercio del siglo XX», en Comas, F.; Motilla, X. y Sureda, B. (eds.): Fotografia i Història de la Educació, Mallorca, Lleonard Muntaner, 20I2, p. 65.

32 Foschi, R.: Op. cit., p. 77.

33 Citado por ibidem, pp. 87-88.

34 Montessori, M.: El método de la Pedagogía científica, aplicado a la educación de la infancia en las Case dei Bambini, Barcelona, Araluce, 1937, 3. ${ }^{a}$ ed., pp. 310-317. Este mismo capítulo en La scoperta del bambino, traducido al catalán en 1984 , tiene dos párrafos más, en uno se insiste en cómo la religión permitirá desvelar un sentido social en las propias acciones y en el párrafo final se señala que estos experimentos se referían a la religión católica, en la que se puede hacer una preparación activa por medio de ejercicios materiales, mientras que esto no era posible en otras religiones que son totalmente ABstractas, por lo que los ejercicios fueron suprimidos. Remite a sus libros sobre el tema: I bambini viventi nella Chiesa, La S. Messa spiegata ai bambini, La vita in Christo, Il libro aperto y el Manuale per la preparazione di un Messale per i bambini. MonTessori, M.: La 
EL MÉTODO MONTESSORI EN LA EDUCACIÓN INFANTIL ESPAÑOLA:

LUCES Y SOMBRAS

CARMEN SANCHIDRIÁN BLANCO

de miel con el catolicismo duró hasta su ruptura con el fascismo y fue sustituida por una cercanía a la teosofía y con la municipalidad republicana de Barcelona ${ }^{35}$.

También colaboró a la difusión de Montessori en España Leonor Serrano, inspectora escolar en Barcelona desde 1913. Pronto vio, según ella cuenta, el problema de la educación de los más pequeños, «olvidados por burócratas» y hasta desdeñados por «maestras sabias», que reclamaba la redención de esas madres y maestras y la redención, el nacimiento espiritual, de la vida psíquica y social de los niños. Leonor Serrano, como Palau, necesitaba una esperanza en un momento, tras el estallido de la I Guerra Mundial, en que todo conducía al pesimismo: «Asistimos a una gran derrota de la cultura, y por lo tanto de la educación. Una espantosa guerra internacional ha venido a probarnos, a nosotros los educadores, la vaciedad, o por lo menos, la insuficiencia de la cultura, no basada en una fortísima educación moral» ${ }^{36}$.

Tras conocerse la obra de Montessori y gracias a los ensayos de este método realizados por parte de Palau Vera; al entusiasmo del secretario del Consejo de Pedagogía de la Mancomunidad de Cataluña, Eladio Homs, y al incondicional apoyo prestado por Hermenegildo Giner de los Ríos desde el Ayuntamiento, se logró reunir un grupo de pensionadas por parte de la Diputación y del mismo Ayuntamiento, por encargo del Ministerio de Instrucción Pública, para acudir al II Curso Internacional Montessori celebrado en Roma en 19I4. Al volver, este grupo creó varias escuelas a la vez que se transformaron otras (públicas y privadas) gracias a una favorable propaganda del método.

La Mancomunidad de Cataluña organizó el III Curso Internacional en 1916, que dirigió Montessori personalmente, pero, a pesar del entusiasmo de sus promotores, este curso no tuvo gran resonancia en España. Creó también un laboratorio de Pedagogía donde ella misma trabajó durante unos tres años, pero, a pesar del empeño de algunos profesores, los vaivenes políticos enfriaron el caldeado ambiente.

Durante los años de esa primera etapa en España, en Barcelona, fue cuando estableció relaciones con el fascismo italiano. En 1923, su hijo escribió a Mussolini desde Barcelona preguntándole cómo era posible que el método montessoriano fuese conocido en el mundo y rechazado en Italia. A partir de ese momento, Mussolini decidió su «refundación» en Italia. Para ello, se puso en marcha en 1924 la Obra Nacional Montessori para difundirlo en Italia y formar maestras bajo el control directo de Montessori. El I Curso se celebró en 1926. En I927 empezó a publicarse L'Idea Montessori; en 1929 se fundó en Roma la Real Escuela de Magisterio del Método donde se enseñaba, de acuerdo con los dictados del régimen, la cultura fascista. La inserción de elementos extraños al método era mal tolerada por Montessori, quien pensaba probablemente «con cierta ingenuidad que podría

descoberta de l'infant, Vic, Eumo, 1984, pp. 329-336. En castellano tenemos La Santa Misa vivida por los niños, Barcelona, Araluce, 1936.

$35 \quad$ Cfr. Foschi, R.: Op. cit., p. 88.

36 Serrano, L.: El método Montessori, Madrid, Publicaciones de la Revista de Pedagogía, I934,

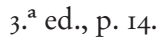


difundir sus principios 'a medida del niño' por medio del fascismo» ${ }^{37}$. En realidad, el fascismo quería explotar la notoriedad de Montessori para difundir sus propios principios y su propia política ${ }^{38}$.

A partir de las denuncias de algunas profesoras de la Escuela hacia otras acusándolas de antifascistas, empezaron a ser espiados también los movimientos de Montessori. Según los espías, desde España, Montessori y su hijo querían mantener buenas relaciones con el régimen fascista enviando de vez en cuando cartas a Mussolini y pidiendo, incluso, su inscripción en el Partido Nacional fascista, pero eran, según este informador, antifascistas y el hijo tenía buenas relaciones con Francesc Macià pues habían organizado escuelas para la Generalitat, para los hijos de obreros y desempleados. En marzo de 1933 se celebró en España la semana Montessori y se dijo entonces que «su modelo está reconocido como el método de la escuela republicana con el apoyo de psicólogos y pedagogos de las Universidades de Barcelona y Madrid ${ }^{39}$. A pesar de esto, se celebró el IV congreso Internacional Montessori en Roma en abril de 1934 donde una espía asistente consideró que la pedagogía montessoriana era extraña «a la ternura austera del régimen» que prepara a los niños «para potenciar la Era fascista consagrada con la sangre heroica de miles de camisas negras ${ }^{40}$.

En 1929, Montessori llegó a Barcelona, reanudó sus actividades como asesora en varios centros para niños (Casa de Maternidad y Expósitos, colegio Montd'Or) y siguió escribiendo. Su amistad con Joan Palau Vera y la fe que este tenía en la filosofía montesoriana dieron como resultado la edición de varios libros, de los que cinco se editaron solo en español y que son hoy prácticamente desconocidos porque se agotaron el mismo año de su publicación y no se han reeditado ${ }^{41}$. Por tanto, este método se implantó en España con garantías de autenticidad ya que ella misma supervisó las primeras realizaciones y alude a ellas en las ediciones sucesivas de su Método de la Pedagogía científica; en I933, había trece escuelas montessorianas en Barcelona y 2r en otras localidades (Cataluña, Castellón y Valencia), pero probablemente habría más que siguieran, al menos parcialmente, su método ${ }^{42}$.

37 Cfr. Foschi, R.: Op. cit., p. 95.

${ }_{38}$ En este vídeo donde puede verse como una niña compone la frase «Benito Mussolini ama molto i bambini»: «Il metodo Montessori presso la Regia Scuola Magistrale Montessori», Giornale LUCE, del 19/6/1935. Archivio Storico LUCE. Disponible en https://www.youtube.com/ watch?v=E2pWbYaTc_o.

39 Foschi, R.: Op. cit., p. 98.

${ }_{40}$ Citado en Foschi, R.: Op. cit., p. 99.

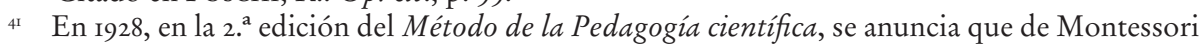
también se habían publicado La Autoeducación en la escuela elemental, Antropología pedagógica, Manual práctico del Método Montessori y Cuaderno de dibujo. En 1937, en la 3. ${ }^{2}$ edición, se anuncian, además, Psico-aritmética, Psico-geometría, La Misa, las prácticas litúrgicas al alcance de los niños y El Niño. Tanto La Autoeducación... como el Cuaderno de dibujo figuran como agotados.

${ }_{42}$ Una buena prueba de la gran difusión que la pedagogía montessoriana había alcanzado en España antes de la Guerra Civil es el comentario que se hace con motivo de la aparición de la obra de XANDRI Y PICH, José: La pedagogía montessoriana (Estudio informativo-crítico sobre "El método de la pedagogía científica»), Madrid, Yagües, 1936. En la recensión de esta obra publicada en el n. ${ }^{\circ}$ I7I 
En 1934, al tener que dejar Italia tras su ruptura con el fascismo y tras el enfriamiento de sus relaciones con la Iglesia católica, se instaló en Barcelona con su hijo ${ }^{43}$. Al estallar la Guerra Civil, salió de España ayudada por el Gobierno británico en uno de sus buques de guerra.

\section{Montessori en la España franquista}

El franquismo es un período largo dentro del cual se consideran básicamente dos etapas, antes y después de 1959, que podemos mantener también al hablar de educación. En el primer período se observa un rechazo de todo lo que tuviera que ver con la Escuela Nueva, con escuela activa y con todo aquello donde apareciera la palabra «libertad», especialmente hasta 1950.

La corriente triunfadora, que no aparece con el franquismo, sino que ya existía antes de la guerra, defendía una escuela «española» frente a las influencias del extranjero. En esos años, como se recoge en la legislación ${ }^{44}$, se insistía en esa españolidad que implicaba el silencio en torno a la Escuela Nueva y el rechazo de todo lo que pudiera recordarla, de manera que la presencia de Montessori era prácticamente nula.

En los años cincuenta, sin embargo, empezaron a aparecer alusiones a Montessori en los Manuales sobre educación de párvulos ${ }^{45}$ y en las historias del pensamiento pedagógico, normalmente junto a Decroly, ya que fueron contemporáneos y ambos estudiaron medicina y llegaron desde ella a la educación especial y de aquí a la educación, en general.

de la Revista de Pedagogía correspondiente a marzo de 1936, se dice que será de alguna utilidad a las maestras de párvulos, «aunque dudemos un poco de la eficacia de estos propósitos de divulgación de una técnica tan sobradamente conocida y ya superada en la actualidad, por otras que, poco a poco, y en una lenta, pero segura transformación, van situando un poco al margen de las actuales exigencias metodológicas en las escuelas de párvulos, la técnica montessoriana». Llega a decir que «el trabajo del señor Xandri es muy estimable, y esperamos que en posteriores intentos ponga su buena voluntad y su cultura profesional al servicio de otras experiencias menos estudiadas que la montessoriana y que representen un progreso respecto a las formas clásicas de organización y trabajo escolares». Se alude a la ya «copiosa bibliografía sobre toda la obra educativa de la doctora italiana». Sin subrayar en el original. Ibidem, pp. I38-139.

43 Solo desde entonces vivió con él de forma continuada, cuando ya tenía unos treinta años. Su hijo se separó poco después y se quedó también aquí con sus hijos Mario y Renilde.

${ }_{44}$ «La etapa republicana de mil novecientos treinta y uno llevó a la Escuela una radical subversión de valores. La legislación de este período puso su mayor empeño en arrancar de cuajo el sentido cristiano de la educación, y la Escuela sufrió una etapa de influencias materialistas y desnacionalizantes que la convirtieron en campo de experimentación de la más torpe política, negadora del ser íntimo de nuestra conciencia histórica», "Preámbulo de la Ley de 17 de julio de 1945 sobre Educación Primaria», BOE, n. ${ }^{\circ}$ 199, I8 de julio de I945, pp. 385-386.

45 Cfr. Maíllo, A.: Manual de educación de pároulos: contestaciones completas al cuestionario de especialización de pároulos de 24 de abril de 1948, Burgos, Hijos de Santiago Rodríguez, 1954, 2. ${ }^{2}$ ed.; Medina, A.: Educación de párvulos, Barcelona, Labor, 1955, 3. . ed. 1967. 
Así, Adolfo Maíllo en su Manual ofrecía las contestaciones completas al cuestionario de especialización de párvulos de 24 de abril de 1948. Solo en el tema $\mathrm{V}$, dedicado a «Estudio especial del sentido del tacto.- El tacto en la Pedagogía montessoriana.- Actitudes correctas» aparece un epígrafe dedicado a Montessori. La O. M. de 20 de octubre de 1953 añadió tres temas al cuestionario oficial de oposiciones a escuelas maternales y de párvulos y la misma editorial publicó entonces un Suplemento al Manual en 1956 incluyéndolos. El primero de ellos es «Parvulistas extranjeros más notables.- Adaptación de sus métodos a la psicología del niño español». Entre los «educadores de párvulos más destacados» se incluye a Froebel, Decroly, Montessori y las señoritas Audemars y Lafendel. Algunos comentarios insisten en la ya citada «españolidad», en que los niños españoles son distintos a los europeos y que, por tanto, no se puede aplicar aquí, sin más, cualquier método. Maíllo indica los rasgos diferenciales del niño español con relación a otros niños europeos que le llevan a decir que «toda prudencia es poca cuando se trata de introducir métodos extranjeros, sobre todo cuando se trate de aquellos que requieren un material y unos ejercicios opuestos a la condición esencial del niño y del hombre españoles: la espontaneidad y la espiritualidad» ${ }^{46}$.

Teniendo en cuenta tales precauciones, justifica su posición relativamente favorable hacia Montessori a partir de la explicación de su religiosidad afirmando que es «un carácter esencial en la pedagogía de María Montessori, italiana de raza, cuyo sentimiento religioso se conjuga maravillosamente con el sentimiento artístico» ${ }^{47}$. En este Manual se dice que la pedagogía de Montessori es la que más importancia ha concedido a los problemas teóricos y prácticos de la educación sensorial: «Ello no es extraño, así como tampoco el carácter científico de esta pedagogía, si tenemos en cuenta los antecedentes y la preparación de la doctora Montessori» ${ }^{48}$.

Otro ejemplo distinto de esa visibilidad de Montessori en los cincuenta lo tenemos al verla incluida en un libro de lectura dirigido a niñas de 9 o Io años. En Cuando las grandes maestras eran niñas ${ }^{49}$. Montessori es uno de los personajes que se ofrecen como ejemplo, se citan algunas de sus aportaciones, su participación en la creación de las Casas de los Niños y se repiten tópicos como que fue la primera mujer que obtuvo en Italia el grado de doctora en Medicina. El autor vincula su dedicación a la pedagogía con el hecho de haber conocido a Maruzella, una compañera de clase «que siempre queda al margen de todo y de todos y que las mira con una mirada vacía y lejana que no se sabe si es de tristeza o de indiferencia. [...] es la última de la clase. La que no ha conseguido nunca aprender la tabla de multiplicar y confunde siempre los nombres de las cosas y de los lugares $»^{50}$.

${ }^{46}$ Maíllo, A.: Op. cit., p. 22.

47 Ibidem, p. I7.

${ }_{48}$ Ibidem, p. 98.

49 Verdaguer, R.: Cuando las grandes maestras eran niñas, Barcelona, Cervantes, 1958, pp. IOI-III.

so Ibidem, p. Ios. 
EL MÉTODO MONTESSORI EN LA EDUCACIÓN INFANTIL ESPAÑOLA:

LUCES Y SOMBRAS

CARMEN SANCHIDRIÁN BLANCO

Esta versión es muy dudosa y no aparece en las biografías que hay sobre ella. Lo más similar puede considerarse un encuentro casual ya cuando estaba estudiando Medicina, citado por Siziare, con un «un niño pequeño sucio y andrajoso, con el que se cruzó en la calle» que «le proporcionó las fuerzas para continuar. [...] absorto en la contemplación de un trozo de papel coloreado, estaba radiante, con una serenidad que estremeció a María» ${ }^{51}$.

No entraremos ahora en describir, analizar y criticar el texto de Verdaguer, pero sí es significativo que haya sido incluida en un libro que se supone que va a hablar de la infancia de los personajes cuando de Montessori se dice que en las múltiples conversaciones que tuvo con sus discípulas apenas contaba nada de su vida privada y que «nunca dejó entrever un recuerdo o una anécdota de sus primeros años $\aleph^{52}$. En esas condiciones, Montessori no era una buena candidata para ser incluida en este libro ya que se llega a afirmar que parecía que su vida hubiera empezado en Roma cuando empezó a estudiar Medicina. La imagen idealizada que se da de ella (como de todos los personajes analizados en esta colección de libros), así como el hecho mismo de su inclusión, son significativos del eco que alcanzó en España en los años finales de su vida.

En esa década de los cincuenta ${ }^{53}$, ya algunos pedagogos obtuvieron becas para viajar al extranjero y en sus visitas se fijaron especialmente en las aplicaciones de los métodos activos y de los procedimientos de la Escuela Nueva. Los docentes que visitaron Italia observaron la aplicación que se hacía del método de Montessori y una maestra acudió especialmente a Perugia para realizar el XXIX Curso Internacional Montessori, logrando una entrevista con ella. Quedó impresionada por su vitalidad personal, pero se mostró crítica con los contenidos del curso, subrayando «desde la simplicidad de las explicaciones hasta la ausencia de discusión y polémica si bien alabó el material y la metodología seguida en la Casa di Bambini» ${ }^{54}$.

Las relaciones de Montessori con el catolicismo fueron importantes desde los comienzos de su carrera profesional y siguieron jugando un papel fundamental hasta sus últimos años de vida. De hecho, gracias a que autores católicos se hicieran eco de su supuesta conversión al catolicismo al final de su vida, fue incluida entre los autores «aprobados» ${ }^{55}$, y eso favoreció que se publicara algún texto suyo y que su hijo visitara Madrid en 1959 para preparar un Curso Internacional Montessori que, según Del Pozo y Braster, no llegó a realizarse ${ }^{56}$. Ella misma reconocía,

sI Sizaire, A.: Op. cit., p. iI.

52 Verdaguer, R.: Op. cit., p. ios.

53 Pozo, M. del y Braster, S.: «El movimiento de la Escuela Nueva en la España franquista (España, 1936-1976): repudio, reconstrucción y recuerdo", Rev. Bras. Hist. Educ., Campinas-SP, I2, n. ${ }^{\circ} 3$ (30) (2012), pp. 15-44. Disponible en http://dx.doi.org/I0.4322/rbhe.20I3.002.

${ }_{4}$ Citado en ibidem, p. 26.

s5 En 1962, el jesuita Pierre Faure acudió a Barcelona para hablar sobre las corrientes «revolucionarias» de la pedagogía contemporánea y destacó las características de la mayoría de los autores, calificando a algunos -como Demolins y Montessori- de «francamente espiritualistas y católicos», y a otros como «ajenos a la mentalidad cristiana», pero también reconoció que «en todos hay muchas luminosas orientaciones, junto con alguna exageración». Citado en ibidem, p. 28.

56 Ibidem, p. 26. 
acercándose así a los católicos, que en el período de entreguerras se había alejado del positivismo y que las pruebas no habían conducido a una pedagogía científica, como parecía indicar el título de las tres primeras ediciones de su Método de la Pedagogía científica. A medida que ella se acercaba a las directrices de la ortodoxia católica, la finalidad espiritual y religiosa iba desplazando las premisas positivistas de partida y los católicos fueron abandonando sus prevenciones contra el método ${ }^{57}$.

A pesar de estos acercamientos a Montessori, hubo que esperar hasta 1956 para que reaparecieran escuelas Montessori en España, como el parvulario dirigido por Antonia Canals, y para que se celebraran cursillos del método para maestras como el que tuvo lugar ese mismo año, representando de nuevo «un cuerpo vertebrado y sistematizado de educación infantil ante las prácticas formalistas y vacías que dominan la enseñanza infantil» ${ }^{58}$. En ese mismo año comenzó la actividad de la Escuela de Jardineras Educadoras que se declaraba heredera de Froebel, Montessori y Decroly.

Hay que destacar la labor de renovación pedagógica realizada desde 1965 por la escuela de maestros Rosa Sensat que condujo a un segundo renacimiento montessoriano promovido por ciertos grupos que impulsaron la creación de escuelas, formación de maestras, etc. En 1966 se celebró la primera Escola d'Estiu de Rosa Sensat, Escola que atrajo durante años a miles de alumnos; partiendo de la metodología de Montessori fueron evolucionando hacia otras más globalizadoras como la de Freinet.

Desde los años setenta siguieron apareciendo escuelas Montessori, cursos sobre su metodología y asociaciones diversas. Así, en 1973 un grupo de profesoras se puso en contacto con la presidenta de la Asociación Montessori Internacional Progresista, Ángeles Martín Aguilera, quien se ofreció para impartir un curso de formación en España y, a lo largo de los tres años siguientes, vino a Madrid cada dos semanas con ese fin. En 1974, Josefina González, amiga y compañera de Ángeles en el colegio Montessori de Londres donde había trabajado is años, volvió a España y, alentada por Ángeles y ante la demanda de un grupo de profesores interesados en organizar de nuevo la Asociación Montessori en España (AME), fue nombrada presidenta, cargo que desempeñó hasta su muerte en 1989. Su objetivo fue propagar y promover los principios psicopedagógicos del Sistema Montessori. Según explican en su web, la AME logró en 2013 el reconocimiento y vinculación a la Asociación Montessori Internacional (AMI) como organismo representante de la misma en España. La AME imparte, desde sus comienzos, cursos de formación a profesores, charlas y coloquios dirigidos a padres y profesionales interesados en la educación.

57 Tras desarrollar el tema del tratamiento del misterio religioso en La Misa vivida por los niños, creció notablemente su aceptación en las escuelas confesionales. Cfr. MONTEssori, M.: La descoberta de l'infant, Vic, Eumo, 1984, p. XXII. Además, la enseñanza religiosa aparece como un capítulo en todas las ediciones, salvo en la primera, de su Pedagogía científica.

58 GonZÁlez-Agàpito, J.: «Educación infantil e industrialización en Cataluña», Historia de la Educación, Salamanca, Io (199I), p. I52. 
EL MÉTODO MONTESSORI EN LA EDUCACIÓN INFANTIL ESPAÑOLA:

LUCES Y SOMBRAS

CARMEN SANCHIDRIÁN BLANCO

\section{Montessori en la España democrática}

Con la llegada de la democracia, la creación de centros Montessori siguió diferentes vías y criterios de forma que no es fácil dar datos fiables ni ofrecer una imagen clara porque Montessori hoy es, en gran medida, una marca y es una marca que vende lo que puede conducir a un uso y abuso de esa marca. Hay quienes llaman a una escuela infantil Montessori como homenaje o para intentar atraer alumnos, pero no porque utilicen el método. Pero lo contrario también es cierto. Se considera a Montessori dentro de la Escuela Nueva, de la Escuela Activa, pero estos términos no se agotan en Montessori, sino que son más amplios, aunque a veces se usan sin precisión. Quizá nunca se haya hablado tanto de Montessori como hoy, lo que no quiere decir que ese término tenga un significado unívoco. Se usa la palabra Montessori o el adjetivo montessoriano para cursos, escuelas, métodos, técnicas abarcando una amplia gama de significados y acentos. Hay quienes lo asimilan con educación no directiva, progresista, educación familiar, ambiente preparado

En un trabajo reciente se habla del número y variedad de las escuelas Montessori en Norteamérica. Creo que esto solo es parcialmente extrapolable a otras zonas, ya que la difusión, al menos nominal, de las escuelas Montessori allí es mucho más fuerte que en nuestro país, donde se ve como un método alternativo $\mathrm{y}$, casi siempre, como moderno, prometedor, alternativo, sin contar con que tiene ya más de cien años.

Hoy hay más de 4.000 escuelas Montessori en EE. UU. y Canadá y unas cien mil en todo el mundo. Ahora bien, la diversidad entre estas escuelas es muy grande, aunque hay quien pueda pensar que «Montessori» es una franquicia y, como tal, las escuelas Montessori, como los McDonald, son siempre parecidas. Esto no es así y en EE. UU. y Canadá hay escuelas Montessori muy diferentes en tamaño, programas, ambiente y servicios ofertados; las hay en bajos de iglesias, en centros comerciales, en barrios y en el centro de las ciudades, en casas en barrios de clase alta y también en barrios obreros y áreas deprimidas; hay escuelas Montessori en el programa Head Start, en guarderías del centro de las ciudades, en campamentos de inmigrantes o en reservas indias. Pero debe haber algo en común en esta diversidad: «A nivel conceptual, las escuelas Montessori comparten una filosofía y un enfoque, pero siempre encontraremos una gran variación enorme entre escuelas que se denominan Montessori ${ }^{59}$. Se reconoce que algunas escuelas Montessori están orgullosas de mantenerse cercanas a los planteamientos originales de Montessori, mientras otras valoran más la flexibilidad y realizan una adaptación pragmática de forma que cada escuela refleja su exclusiva combinación de instalaciones, programas, personalidad e interpretación de la visión de Montessori» ${ }^{60}$.

59 Montessori Ior: Some basic Information that Every Montessori Parent Should Know. Tim Seldin, President The Montessori Foundation. http://www.montessori.org/sitefiles/montessori_ioI_nonprintable.pdf.

6० Idem. 
Esto no se refiere concretamente al caso español, pero si aquí analizásemos las diversas instituciones Montessori también encontraríamos grandes diferencias, especialmente entre los grandes centros Montessori -los que abarcan desde la educación infantil hasta el bachiller-y muchas escuelas infantiles que usan su nombre. Por otra parte, el entramado de asociaciones que llevan hoy en día el nombre Montessori es realmente complejo y merecería, por sí solo, un análisis más detallado. Citaremos alguna, sin pretender hacer un listado exhaustivo. En primer lugar, la AMI, ya citada, fundada por Montessori en 1929. Ella fue su presidenta hasta su muerte, y también han sido presidentes de la misma su hijo Mario y su nieta Renilde (fallecida en 20I2). En Estados Unidos, existe también la American Montessori Society (AMS). En una reciente publicación se indicaba que había 33 sociedades afiliadas a la AMI «y muchas más siguen creándose en la actualidad», lo cual indica indefinición en los datos ${ }^{61}$.

Además de esta asociación, simplemente a modo de muestra y en una rápida búsqueda, existen, entre otras:

- American Montessori Society (AMS)

- International Montessori Society (IMS)

- Montessori Center International (MCI)

- Montessori Accreditation Council for Teacher Education (MACTE) Organización que acredita la formación de guías Montessori

- Montessori Institute of America (MIA)

- Montessori Educational Programs International (MEPI)

- Montessori World Educational Institute (MWEI)

- National Center for Montessori Education (NCME)

Además, hay muchas asociaciones nacionales, por ejemplo, británica, española, mexicana, australiana, etc. Y hay asociaciones locales como la Asociación Montessori de Málaga ${ }^{62}$ o la Asociación Montessori del Sur de España. Esta variedad de asociaciones implica una dificultad importante a la hora de marcar las pautas acerca de la denominación «Montessori» de una escuela, entre otros motivos porque hay escuelas que llevan el nombre como «homenaje» a Montessori, pero que no siguen su método (lo mismo ocurre con colegios que se llaman, por ejemplo, Andrés Manjón y no tienen nada que ver con las Escuelas del Ave María).

Para conocer realmente la difusión del método Montessori hoy en nuestras aulas de infantil (su aplicación en primaria y secundaria es mucho menor, aunque hay algunos centros) hay cuatro elementos, según la AME, que no deben faltar:

- Mezcla de 3 o más edades en las aulas.

- Disponibilidad y uso de materiales Montessori para el aprendizaje.

- Maestros/as formados/as específicamente en el método Montessori.

${ }^{6}$ Poussin, Ch.: La pedagogía Montessori: Una Introducción al método que revolucionó la enseñanza, Buenos Aires, Siglo XXI, 2019,

62 https://asociacionmontessori-malaga.weebly.com/. 
EL MÉTODO MONTESSORI EN LA EDUCACIÓN INFANTIL ESPAÑOLA:

LUCES Y SOMBRAS

CARMEN SANCHIDRIÁN BLANCO

- Libertad de movimiento de los alumnos en las aulas que les permita escoger las actividades a realizar guiados por su propia motivación ${ }^{63}$.

Según la web de la AME, hay 24 colegios Montessori en España (se pueden ver los incluidos en cada Comunidad Autónoma), pero es evidente que la lista es mayor y que el número dependerá del criterio. En Andalucía, por ejemplo, figuran cuatro (en Almería, Córdoba, Marbella y Sevilla), pero con una búsqueda sencilla en Internet por provincias aparecen varios más. Buscando en las webs de algunos de los centros, vemos datos interesantes. Por ejemplo, el Montessori School de Madrid, que figura en el listado de la MAE, es un colegio británico en el que, según indican en su web,

Durante el ciclo de educación infantil (de 0 a 5 años), los niños siguen un programa de enseñanza en inglés cuyos objetivos están centrados en el programa de «Early Years» del sistema británico, junto con una metodología y actividades adaptadas del sistema Montessori. [...]. Empezamos hace ya más de 20 años con nuestra primera escuela de infantil y primaria, siempre priorizando la Introducción de los métodos de María Montessori dentro del contexto de un colegio inglés en España ${ }^{64}$.

Sería interesante conocer las escuelas o colegios Montessori que hay hoy en España, pero no hay datos fiables. Habría que indicar un criterio que podría ser el cumplimiento de los cuatro elementos antes mencionados. Contamos con la información que los centros que se autodenominan Montessori nos ofrecen en sus páginas web y con algunos listados, sin pretensión de exhaustividad, donde se incluyen datos a partir de los cuales buscar. Hay una web que ofrece un «Listado de proyectos de Pedagogía alternativa en España ${ }^{65}$ donde hay datos por provincia o Comunidad Autónoma de centros incluidos en alguna de estas nueve categorías (algunas poco claras): Libre/Viva, Montessori, Waldorf, Enfoque Reggio-Emilia, Amara-Berri, Educación democrática, Escuela-Bosque, Comunidad Aprendizaje y Otras/Varias. Buscando Montessori en Madrid, aparecen 2i centros, que acogen desde bebés hasta distintas edades; todos ellos, o tienen Montessori en su nombre o aluden a ella en su presentación ${ }^{66}$. Entre ellos, vemos muchos que se anuncian como colegios bilingües o trilingües: uno se anuncia como «el primer centro infantil que combina el concepto chino de educación temprana con métodos occidentales de probada eficacia en el aprendizaje: el método Montessori junto con el sistema de disciplina positiva»; otro dice que «cuenta con un proyecto educativo [...] configurado bajo los pilares de neurociencia educativa, pedagogía Montessori y American Education»; uno indica que es un espacio de juego en la naturaleza: «basado en el libre desarrollo del niñ@, todas las emociones son válidas, aceptadas y acompañadas, usamos material Montessori; se realizan otras ac-

${ }_{3}$ http://www.metodomontessori.com/montessori-en-espana/panorama-espanol.html.

${ }_{64}$ http://www.montessorischool.es/.

6s http://ludus.org.es/es/projects.

${ }_{66}$ https://ludus.org.es/es/projects?province_id=4I\&age=admits_children\&pedagogy_id=3. 
tividades complementarias: grupos de juego libre, juegos en la naturaleza, talleres familiares», y así sucesivamente. En la provincia de Málaga figuran cuatro: tres colegios para niños de hasta I2 años, Beworldwise, Centro Internacional Maria Montessori e International Montessorischool Caracoliris y un espacio para niños de cero a seis.

Algunos de los centros Montessori privados indican que se hace una selección de sus alumnos y este es un punto importante a tratar sobre todo cuando se quiere hablar de los resultados, de la eficacia de un método o compararlo con otros métodos. Se ha intentado en los últimos años demostrar científicamente que un método educativo es mejor que los demás (este sería el sueño de todos los creadores de un método), pero los resultados no pueden considerarse definitivos porque había variables no controladas y porque el concepto mismo de resultados mejores es relativo. Desde mediados de los sesenta se han hecho investigaciones comparando el método Montessori con otros buscando evidencias científicas que permitan defender las ventajas de este método frente a otros, con todas las dificultades, o incluso con la imposibilidad ya sabida, que esto implica. La primera fue el Cincinnati Montessori Research Project en 1964 y recibió críticas por los tests utilizados y por problemas de interpretación: ¿qué medimos y qué queremos medir?

En 2006, en la revista Science dos psicólogas publicaron un estudio comparando Montessori con otras escuelas de Milwaukee ${ }^{67}$, pero se señalaba la conveniencia de replicar su investigación en otras escuelas Montessori dado que estas pueden variar mucho. La escuela implicada en este caso estaba afiliada a AMI/ USA, lo cual implica que la implementación del método era tradicional y fiel. Según sus resultados, si el método se aplica estrictamente, la educación Montessori proporciona habilidades académicas y sociales iguales o superiores a las ofrecidas por un conjunto de otro tipo de escuelas ${ }^{68}$. Como vemos, frente a la flexibilidad a la que se alude a la hora de hablar de las distintas escuelas Montessori, cuando se quiere comparar resultados entre distintas escuelas, la aplicación estricta del método es un requisito previo y sine qua non para abordarlo.

Así Montessori entra en el debate científico, cien años después de haber publicado su Método de la Pedagogía científica. Es lógico que las escuelas Montessori defiendan que los resultados de sus alumnos son mejores. Sin embargo, es preciso controlar otras variables que puedan influir tanto como el método seguido en las clases ya que lo que sí parece probado, en cualquier contexto, es que la calidad de

${ }^{67}$ Lillard, L. y Else_Quest, N.: «The Early Years: Evaluating Montessori Education», Science, 313, I893 (2006), pp. I893-I894. DOI: I0.II26/science.II32362 http://faculty.virginia.edu/ASLillard/PDFs/Lillard\% 20\&\% 2oElse-Quest\% 20(2006).pdf.

${ }_{68}$ «It would be useful to replicate these findings in different Montessori schools, which can vary widely. The school involved here was affiliated with AMI/USA, which has a traditional and relatively strict implementation. At least when strictly implemented, Montessori education fosters social an academic skills that are equal or superior to those fostered by a pool of other types of schools». Ibidem, p. I894. 
EL MÉTODO MONTESSORI EN LA EDUCACIÓN INFANTIL ESPAÑOLA:

LUCES Y SOMBRAS

CARMEN SANCHIDRIÁN BLANCO

las interacciones con los padres es el mejor predictor de los resultados escolares ${ }^{69}$. De acuerdo con esto, hay que valorar el peso relativo de la educación familiar y de los métodos pedagógicos y la interacción entre ambos contextos ${ }^{70}$.

Hoy el método Montessori, entre ciertos grupos sociales, se percibe como alternativo, progresista. En algunos sitios, EE. UU. sobre todo, está vinculado al movimiento de escolarización en casa, homeschooling, y se ofrecen webs donde se indica la posibilidad de sustituir los materiales Montessori por otros más baratos o hechos en casa, lo que choca con los principios de la $\mathrm{AMI}^{71}$; dónde hacerse con los materiales; cómo organizar la escuela en casa, etc. Otros grupos lo eligen por considerarlo elitista, un signo de distinción, por estar de moda, por ser moderno, por no agobiar a los niños, etc. Algunos partidarios del colecho ${ }^{72}$ se acercan a este método. Es evidente que estas razones, por sí solas, como tantas otras, tienen poco que ver con el método Montessori. O sí, pero dependerá de qué se haga y cómo se haga.

Se celebró en 2014 el centenario de la primera escuela Montessori en España, en 2015 el de la publicación en castellano de su Pedagogía científica y en 2020 el I50 aniversario de su nacimiento. Quizá sea un buen momento para revisar los resultados de sus experiencias y de sus intuiciones pedagógicas a la luz de lo que hoy sabemos sobre educación y del ser humano como sujeto bio-psico-social.

\section{Conclusiones}

Montessori ha dejado en España un poso visible en las nuevas tendencias y la manera actual de entender la educación infantil, aunque hay aspectos de esta que se le atribuyen cuando en realidad son fruto de esos años y no se le pueden atribuir específicamente a ella.

No se puede negar la relevancia de Montessori y la importancia de sus aportaciones, pero hay que acercarse a su pensamiento sin prejuicios. Hay que desmi-

69 Foschi, R.: Op. cit., 154

70 K. Dohrmann, en 2003, presentaba «Outcomes for Students in a Montessori Program, A Longitudinal Study of the Experience in the Milwaukee Public Schools» donde afirmaba que «This study supports the hypothesis that Montessori education has a positive long-term impact. Additionally, it provides an affirmative answer to questions about whether Montessori students will be successful in traditional schools». http://www.montessori-ami.org/research/outcomes.pdf. En este documento se citan algunas investigaciones que demuestran que los alumnos «Montessori» son mejores en ciertos aspectos, aunque a los I2 años las diferencias se han atenuado. Otro de los aspectos que ha atraído la atención de los investigadores es el paso y el rendimiento de alumnos que han empezado su escolaridad según el método Montessori a otros métodos o a escuelas más convencionales. http://montessori.org.au/research/MontessoriValidatedByResearch.pdf.

${ }_{71}$ La AMI en Estados Unidos (AMI/USA) mantiene que cada aula debe estar equipada con un juego completo de materiales Montessori realizados por un fabricante autorizado por la AMI: The Juliana Group, Inc. (Gonzagarredi) o Nienhuis Montessori USA.

${ }_{72}$ Montessori no dijo nada del colecho, pero en los blogs sobre Montessori aparece el tema. Por ejemplo: https://www.montessoriencasa.es/colecho-y-montessori/ https://www.recursosmontessori.com/colecho/colecho-en-la-filosofia-montessori [consultadas I4-5-2020]. 


\section{CARMEN SANCHIDRIÁN BLANCO}

tificar a los ídolos, como se ha hecho, por ejemplo, recientemente con Decroly, con quien tantos puntos en común tenía, aunque ninguno de los dos quisiera admitirlo $^{73}$. Hoy Montessori es una marca y, como toda marca, tiene su significado para quien la usa y para los que ven que alguien la usa. El problema es elegir un colegio como «distintivo social» ${ }^{74}$.

Montessori ha sido una figura controvertida desde sus primeros años de actividad pública. Ha sido alabada, criticada, venerada, despreciada... Empezó las Casas de los Niños con Talamo y poco después terminaron mal. Tuvo relaciones con las hermanas Franciscanas Misioneras de María y tampoco terminaron bien. Con la Iglesia católica sus relaciones experimentaron frecuentes altibajos. Con Mussolini también tuvo años de buena relación hasta que decidió romper esa relación. En los EE. UU., cuando fue por primera vez tuvo como mano derecha a Helen Parkhurst con la que pronto rompió y esta publicó su propio método, el Plan Dalton ${ }^{75}$.

Su vida, el hecho de ser madre soltera, su relación con su hijo y sus nietos, todo ello estuvo rodeado de un halo de misterio y de mitificación que dificulta ver a la persona y sus aportaciones. El acceso a los documentos de la AMI en Ámsterdam también es restringido y el hecho de que su nombre sea sinónimo de «progresismo», «libertad», «innovación», etc., hace que sea difícil no solo saber las escuelas Montessori que hay hoy en España, o en otros países, sino también saber cómo se aplica el método: el uso de los materiales, la formación del personal, la organización del espacio, las actividades de la vida diaria, la educación de niños de varias edades juntos, etc.

73 Depaepe, M.; Simon, F. y Van Gorp, A.: «The Canonization of Ovide Decroly as a 'Saint' of the New Education", History of Education Quarterly, 43-2 (2003), pp. 224-249 y VAN GoRP, A.; Simon, F. y Depaepe, M.: «Introducción», en Decroly, O.: La función de globalización y la enseñanza, y otros ensayos, Madrid, Ed. Biblioteca Nueva/Ministerio de Educación y Ciencia, 2006. El hecho de que Montessori sobreviviera a Decroly casi veinte años debe tenerse en cuenta al estudiar los procesos de canonización de ambos, de su conversión en héroes. Decroly no creó una red similar a la de Montessori (fabricantes, asociación, titulaciones, etc.). La rivalidad entre los seguidores de ambos era latente. Cfr. VAN Gorp, A.: «Ovide Decroly (2006). A Hero of Education», en Smeyers, P. y Depaepe, M. (eds.): Educational Research: Why 'What Works' Doesn't, Dordrecht, Springer, 2006, pp. 38 y 4I-42.

${ }_{74}$ El interés por las escuelas Montessori subió un $65 \%$ en Inglaterra cuando se hizo público que el príncipe Jorge iba a asistir a una de ellas. OTERo, B.: «El príncipe Jorge aumenta la fama del método Montessori», El País, 8 de febrero de 2016. https://elpais.com/elpais/2016/o2/o5/estilo/I454691773_405553.html.

${ }_{75}$ Parkhurst se unió a Montessori en Nueva York y durante un tiempo fue la directora de todas las escuelas Montessori en Estados Unidos. Luego, Parkhurst empezó a crear sus propias escuelas y a publicar sus propias ideas en artículos y libros. Introdujo su Plan Dalton en I9I4 en una escuela privada, Children's University School (hoy The Dalton School en Nueva York), buscando el equilibrio entre las capacidades de los niños y las necesidades de la comunidad. Para ella los niños aprenden más eficazmente en un entorno que les permita libertad de elección y seguir su propio ritmo ya que el aprendizaje es un proceso rigurosamente individual. En vez de asignaturas, plantea la idea de contratos de aprendizaje: «Me comprometo a aprender....». PArkhurst, H.: Education on the Dalton Plan, London, Bell, 1924. 
EL MÉTODO MONTESSORI EN LA EDUCACIÓN INFANTIL ESPAÑOLA:

CARMEN SANCHIDRIÁN BLANCO

En general, podemos decir que los métodos se adoptan en cada país y, una vez adoptados, son adaptados. La difusión, recepción y aplicación de estos métodos, o de variantes de los mismos, en distintos países y momentos, así como el papel que han jugado en todo ello ideologías políticas y religiosas, y más aún en el caso de Montessori, sigue siendo una tarea en parte pendiente porque es importante conocer y leer a Montessori y, probablemente, convertir algunos de sus principios en nucleares en nuestra educación, aunque no sea en aulas Montessori. Del mismo modo que hoy muchos de sus principios están asimilados, forman parte de nuestra educación infantil sin saber que fue ella quien los planteó, la incorporación de otros también podría ser positiva para nuestra educación infantil, manteniendo al mismo tiempo la diversidad y pluralidad entre los centros, pero esto no es lo que se espera de un colegio autodenominado Montessori.

La metodología aplicada en las escuelas Montessori ha ido cambiando desde las primeras Casas de los Niños, pero su teoría se mantiene. Los planteamientos de Montessori que mantienen hoy plena vigencia son, básicamente, los teóricos: centrarse en el niño como ser humano en proceso de desarrollo, su defensa del aprender haciendo y de la autonomía personal, subrayar la importancia del ambiente y de la concentración para aprender, su concepto de educación que va más allá de la acumulación de contenidos, y considerar la actividad motriz y los ejercicios sensoriales como fuentes básicas para el aprendizaje y el desarrollo. Sin embargo, tanto las actividades concretas que proponía para adquirir conocimientos en las distintas áreas de desarrollo y aprendizaje como el material han ido siendo revisados y modificados de acuerdo con los conocimientos y recursos con que hoy contamos o incorporados como algo habitual, ya sin etiqueta. Quizá en vez de decir qué es Montessori, habría que decir qué no es para desmontar algunos estereotipos creados en torno a ella que se repiten como eslóganes: no es aprender jugando, no es que el niño haga lo que quiera, no es aprender sin esfuerzo... Sí es disfrutar con lo que se elige hacer (dentro de ciertas opciones) sin necesidad de recompensas externas, concentrados en las tareas, es dar importancia al trabajo individual y a la autodisciplina, etc.

Más de cien años después, la actualidad del «método Montessori», o de algunos de los «principios teóricos sobre los que se basa su método», es posible gracias a su capacidad de adaptación; sin embargo, en esto, como en todo, mayor capacidad de adaptación implica menor fidelidad. Solo con un acercamiento serio a su obra podrá esta interpretarse, conocerse y valorarse realmente; del mismo modo, hay que estudiar con detalle las instituciones que llevan el nombre de Montessori para poder discriminar lo que es realmente Montessori, porque se siguen sus principios pedagógicos, de lo que es una mera utilización de un nombre o de unos materiales. 
